This is the post-print copy (i.e. final draft post-refereeing) of a manuscript submitted to the journal Food Chemistry (Elsevier, ISSN: 0308-8146)

Please cite as:

Cao H, Högger P, Arroo R, Xiao J (2020) Flavonols with a catechol or pyrogallol substitution pattern on ring B readily form stable dimers in phosphate buffered saline at four degrees Celsius. Food Chemistry 311:

https://doi.org/10.1016/j.foodchem.2019.125902 


\title{
Flavonols with a catechol or pyrogallol substitution pattern on ring B readily form stable dimers in phosphate buffered saline at four degrees Celsius
}

\author{
Hui Cao ${ }^{\mathrm{a}}$, Petra Högger ${ }^{\mathrm{b}}$, Randolph Arroo $^{\mathrm{c}}$, Jianbo Xiao ${ }^{\mathrm{d}, *}$ \\ ${ }^{a}$ College of Food Science, Fujian Agriculture and Forestry University, Fuzhou, Fujian 350002, China \\ ${ }^{\mathrm{b}}$ Institut für Pharmazie und Lebensmittelchemie, Universität Würzburg, 97074 Würzburg, Germany \\ ${ }^{c}$ De Montfort University - Leicester School of Pharmacy, The Gateway, Leicester LE1 9BH, UK \\ ${ }^{\mathrm{d}}$ Institute of Food Safety and Nutrition, Jinan University, Guangzhou, China \\ *Corresponding author, E-mail address: jianboxiao@yahoo.com
}

\begin{abstract}
Phosphate buffered saline (PBS) is a buffer commonly used in biological research. The stability of a series of flavonoids, i.e. myricetin, kaempferol, baicalein, luteolin and quercetin in PBS was assessed. Apigenin proved very stable when incubated with PBS and was used as a control. Kaempferol and baicalein were less stable, and small amounts of oxidized and hydroxylated products could be detected. Flavonols with catechol or pyrogallol structure in ring B are unstable in PBS $(\mathrm{pH}=7.4)$ at $4{ }^{\circ} \mathrm{C}$ and were converted into their stable dimers and oxidized products within 5 seconds. The chosen experimental conditions improved the stability of dimers and allowed their detection.
\end{abstract}

Keywords: phosphate buffered saline; myricetin; quercetin; luteolin; dimerization; stability; oxidation 


\section{Introduction}

Flavonoids are important dietary polyphenols and abundantly present in fruits, vegetables, green tea, red wine, cocoa, and related food products. Flavonoids play a number of crucial roles in plants. For example, they are involved in stress tolerance with consequent contribution to crop productivity (D'Amelia et al., 2018), they control key steps of cell growth and differentiation (Agati et al., 2012), and regulate developmental processes in the whole plant and in individual organs (Brunetti et al., 2013).

Flavonoids possess various pharmacological and physiological functions which are commonly related to their antioxidant potential (Cao et al., 2018; Zhao et al., 2019; Chen et al., 2018a and 2018b; Xiao and Högger, 2015a). The most abundant naturally occurring flavonoids contain one or more hydroxyl groups attached to rings A and B. These functional groups increase the reactivity, and consequently reduce stability of these compounds (Xiao, 2018; Xiao and Högger, 2015b). Flavonoids with a pyrogallol or catechol group are easiest to oxidize because the initially formed phenoxyl semiquinone radical can be stabilized by a second oxygen atom (Waterhouse \& Laurie, 2006).

The structure-stability of flavonoids in methanol and Dulbecco's modified Eagle's medium (DMEM) have been investigated (Xiao and Högger, 2015b). Flavonoids are very stable in methanol at $37^{\circ} \mathrm{C}$. However, flavonoids with catechol or pyrogallol structure were evidently instable in DMEM (Xiao and Högger, 2015b), e.g. myricetin completely disappeared within 10 minutes. The amounts of oxidized products of quercetin and luteolin steadily increased with increasing exposure time to atmospheric oxygen (Ramešová et al., 2012).

The oxidation of flavonols has been detected in buffers at different $\mathrm{pHs}$ under anaerobic conditions. Both the oxidation potential and the number of electrons and protons involved in the oxidation 
strongly depend on the level of dissociation of flavonol in solution (Sokolová et al., 2011; Nematollahi and Malakzadeh, 2003). Two-electron and two-proton oxidation processes happen in acidic solutions, whereas one-electron oxidative processes take place in alkaline conditions (Dangles, Fargeix, Dufour, 1999; Ramešová, Degano, Sokolová, 2017). The different chemical structures of flavonoids significantly affect the oxidation process and products formed (Sokolová et al, 2016); whereas flavonols are oxidized to form a benzofuranone derivative and several open structures, no benzofuranone derivatives are formed after oxidation of flavones (Sokolová et al, 2016). Alkaline conditions and the presence of oxygen stimulate oxidation of flavonoids.

Although dimerization of flavonoids has been reported, it was difficult to characterize dimers due to their instability in the presence of air (Sokolová et al., 2012 and 2016). We dissolved flavonols in phosphate buffered saline (PBS) $(\mathrm{pH}=7.4)$ at $4{ }^{\circ} \mathrm{C}$ which improves the stability of dimers and allows their detection. PBS is a buffer commonly used in biological research. It is isotonic and non-toxic to cells, and can be used to rinse vials containing cells. Herein, the products that were formed within 5 seconds from unstable flavonoids with a catechol or pyrogallol substitution in ring B, i.e. myricetin, kaempferol, baicalein, luteolin and quercetin, were assessed in PBS at 4. Apigenin, which is stable when incubated with PBS was used a control.

\section{Materials and methods}

\subsection{Chemicals and reagents}

Myricetin (>97.0\%), kaempferol (>97.0\%), baicalein (>98.0\%), and apigenin (>98.0\%) were purchased from Tokyo Chemical Industry Co., Ltd. (Shanghai, China). Luteolin (>98.0\%) and quercetin (>97.0\%) were obtained from Aladdin Chemistry Co. Ltd. (Shanghai, China). HPLC grade methanol, acetonitrile, and DMSO were purchased from Merck (Darmstadt, Germany). 
HyClone PBS solution (1×, $0.0067 \mathrm{M} \mathrm{PO}_{4}$, without calcium and magnesium) was purchased from GE Healthcare Life Sciences (UK). Deionized water was prepared using a Milli-Q Integral water purification system (Millipore, Bedford, MA, USA). Methanol and acetonitrile are of HPLC grade.

\subsection{Preparation of flavonoids stock solutions}

Individual flavonoids were dissolved in DMSO to obtain standard stock solutions $\left(5 \times 10^{-3} \mathrm{~mol} / \mathrm{L}\right)$. These standard stock solutions were kept at $-20^{\circ} \mathrm{C}$.

\subsection{Incubation of flavonoids with PBS}

Ten microliter of a flavonoid stock solution $\left(5 \times 10^{-3} \mathrm{~mol} / \mathrm{L}\right)$ was added to $190 \mu \mathrm{L}$ PBS $\left(4{ }^{\circ} \mathrm{C}\right)$ in a $1.5-\mathrm{mL}$ centrifugal tube. Immediately, the tube was inverted for 5 times. Then, $200 \mu \mathrm{L}$ methanol $\left(-20^{\circ} \mathrm{C}\right)$ was added to the tube to block the reaction. After centrifugation at $12,000 \times \mathrm{g}$ for $15 \mathrm{~min}$ at $0{ }^{\circ} \mathrm{C}$, a $5.0 \mu \mathrm{L}$ aliquot was injected to the UPLC-MS-MS system.

\subsection{Incubation of flavonoids with methanol}

Ten microliters of a flavonoid stock solution $\left(5 \times 10^{-3} \mathrm{~mol} / \mathrm{L}\right)$ was added to $190 \mu \mathrm{L}$ methanol (4 $\left.{ }^{\circ} \mathrm{C}\right)$ in a $1.5-\mathrm{mL}$ centrifugal tube. Immediately, the tube was inverted for 5 times. Then, $200 \mu \mathrm{L}$ methanol $\left(-20^{\circ} \mathrm{C}\right)$ was added to the tube to block the reaction. After centrifugation at $12000 \times \mathrm{g}$ for $15 \mathrm{~min}$ at $0{ }^{\circ} \mathrm{C}$, a $5.0 \mu \mathrm{L}$ aliquot was injected to the UPLC-LTQ Orbitrap MS-MS system.

\subsection{UPLC-LTQ Orbitrap mass analysis}

In qualitative analysis, the assay was performed using an ultimate 3000 hyperbaric liquid chromatography system coupled to a LTQ Orbitrap mass spectrometer via an ESI interface. The 
chromatography system consisted of an auto-sampler, a diode array detector, a column compartment and two pumps. Xcalibur, Metworks and Mass Frontier 7.0 software package were used for data collection and data analysis. Liquid chromatographic separations were performed by a Waters ACQUITY UPLC HSS T3 column $(2.1 \times 150 \mathrm{~mm}, 1.8 \mu \mathrm{m})$. The mobile phase consisted of $0.1 \%$ formic acid in water (solvent A) and acetonitrile (solvent B). The samples were eluted with the following linear gradient: 5-10\% B at 0-6 min, $10-25 \%$ B at 6-30 min, 25-35\% B at 30$35 \mathrm{~min}, 35-40 \% \mathrm{~B}$ at $35-40 \mathrm{~min}, 40 \% \mathrm{~B}$ at $40-45 \mathrm{~min}, 40-5 \% \mathrm{~B}$ at $45-50 \mathrm{~min}$. The flow rate was $0.30 \mathrm{~mL} / \mathrm{min}$. The injection volume was $5.0 \mu \mathrm{L}$. The temperature-controlled column oven was set at $30^{\circ} \mathrm{C}$ and the sampler was set at $8{ }^{\circ} \mathrm{C}$. The ESI source parameters were as follows: Both positive and negative ionization modes were used in the analysis. For positive mode, the capillary temperature was $350{ }^{\circ} \mathrm{C}$, sheath gas $\left(\mathrm{N}_{2}\right)$ flow rate was $40 \mathrm{psi}$ and aux gas flow rate was $10 \mathrm{psi}$, ion spray voltage was set at $3.5 \mathrm{kv}$. While for negative mode, the capillary temperature was $350{ }^{\circ} \mathrm{C}$,

sheath gas flow rate was $35 \mathrm{psi}$, and aux gas flow rate was $10 \mathrm{psi}$, ion spray voltage was set at -3.2 $\mathrm{kv}$. In the FT cell, full MS scans were acquired in the range of $\mathrm{m} / \mathrm{z}$ 50-1500 with a mass resolution of 30,000. The MS-MS experiments were set as data dependent scan.

\section{Results and discussion}

\subsection{Apigenin}

As shown in Figure 1, apigenin was very stable in the current experimental conditions, showing a similar TIC chromatography to that obtained in methanol. These results are in agreement with previous observations that found apigenin very stable during $3 \mathrm{~h}$ incubation in DMEM at $37^{\circ} \mathrm{C}$ with $5 \% \mathrm{CO}_{2}$ and incubated with PC-3 cells in RPMI 1640 medium containing $10 \% \mathrm{FBS}$ at $37^{\circ} \mathrm{C}$ with 5\% $\mathrm{CO}_{2}$ for $20 \mathrm{~h}$, respectively (Xiao and Högger, 2015b; Sharma et al., 2014). However, 
although in the present work no dimers of apigenin could be detected in neutral PBS $(\mathrm{pH}=7.4)$, in alkaline potassium ferricyanide oxidative coupling of apigenin resulted in two dimers identified as as 3,3- and 3,3'-biapigeninyl (Molyneux, Waiss Jr. \& Haddon, 1970). Thus, correct reaction conditions are essential when monitoring in the dimerization process.

\subsection{Baicalein}

Baicalin in PBS proved fairly stable. Its main oxidation product, baicalein quinone $(\mathrm{m} / \mathrm{z}=270.2508)$, was formed in very low amounts (Figure 2$)$. In addition, baicalein derivatives $(\mathrm{m} / \mathrm{z}=287.05$ and 303.05$)$ with one or two more hydroxyl groups were detected. The oxidation of baicalein proceeds via two electron transfer steps where hydroxyl groups at C-6 and C-7 of baicalein were oxidized to form an $o$-benzoquinone (Zhu et al., 2004). Then, a hydroxylation can take place to form hydroxylated baicalein at position C-3 (Ramešová et al., 2013).

\subsection{Kaempferol}

When kaempferol was added to PBS, a new small peak with retention time of 37.86 min occurred

(Figure 3). UPLC-MS-MS analysis of this new product showed an M+1 peak at $\mathrm{m} / \mathrm{z} 301.1068$ upon which the product was identified as a quercetin quinone derivative $(\mathrm{mw}=300.1068)$, present in very small amounts. Some kaempferol was oxidized to further hydroxylated derivatives in PBS. Oxidative degradation of kaempferol at $\mathrm{pH} \quad 8$ resulted in the formation of 4hydroxyphenylglyoxylic acid and phloroglucinolcarboxylic acid (Takahama, 1987). In current study, after inverting the tube five times, kaempferol proved stable and only very little kaempferol was oxidized. 


\subsection{Luteolin}

When luteolin was added to PBS, several new compounds occurred within 5 seconds when compared with methanol. Three peaks appear in the TIC chromatography at $36.29,36.68$, and $37.40 \mathrm{~min}$ (Figure 4a) with $\mathrm{m} / \mathrm{z}$ of 571.3125, 571.2929, and 571.2768, respectively. LC-MS-MS analyses revealed that these new products correspond to luteolin dimers (Supplementary 1). The main ion peaks (+ESI) of luteolin dimer $1\left(\mathrm{R}_{\mathrm{t}}=36.26 \mathrm{~min}\right)$ at $\mathrm{m} / \mathrm{z}$ values are: $571.31250,529.33911$, 435.21484, 419.21191 (100\%), 393.23334, and 287.13531 (Figure 4b). The main ion peaks (+ESI) of luteolin dimer $2\left(\mathrm{R}_{\mathrm{t}}=36.72 \mathrm{~min}\right)$ at $\mathrm{m} / \mathrm{z}$ values are: 571.29291, 529.22821, 445.211545, 419.22223 (100\%), 393.20819, and 287.13531 (Figure 4c). The main ion peaks (+ESI) of luteolin dimer $3\left(\mathrm{R}_{\mathrm{t}}=37.36 \mathrm{~min}\right)$ at $\mathrm{m} / \mathrm{z}$ values are: $553.26447463 .04510,445.20038,419.16223$, and 287.13580 (Figure 4d). A dimer of luteolin formed in $3.6 \times 10^{-3} \mathrm{M} \mathrm{KOH}$ was observed previously in very small amounts (Sokolová et al., 2016). The main peaks of that dimer at $\mathrm{m} / \mathrm{z}$ values are 569 , 417, 285, and 151 in negative mode (Sokolová et al., 2016), which is similar to luteolin dimers in the current study. Luteolin tends to be oxidized in $0.1 \mathrm{~m} \mathrm{KCl}-3.6 \times 10^{-3} \mathrm{M} \mathrm{KOH}$ solution and aqueous solution during exposure to atmospheric oxygen (Ramešová et al., 2012 and 2013). Luteolin was seen to be first oxidized to quinone, followed by hydroxylation in aqueous solution (Ramešová et al., 2012 and 2013). However, the authors did not detect any dimers of luteolin. Sokolová et al. (2016) investigated the decomposition of luteolin in slightly alkaline solution under ambient conditions from 10 min to several hours. Luteolin were oxidized to their hydroxylated derivatives and typical open structures (Sokolová et al., 2016). 


\subsection{Quercetin}

When quercetin was added to PBS, compared with that in methanol, immediately a new main product with retention time of 44.85 min was formed (Figure 5a). UPLC-ESI-MS/MS analysis of this new product reveals a main peak with $\mathrm{m} / \mathrm{z}$ of $603.0744(\mathrm{M}+1)$ in positive mode. The main ion peaks (+ESI) at m/z values are: $585.23206(100 \%), 567.21735,557.25079,433.11322,313.05121$, 303.05902, and 273.05591 (Figure 5b). The new product was identified as the dimer of quercetin quinone (Pham, Bortolazzo \& White, 2012). Quercetin was rapidly oxidized in PBS and formed a dimer in fewer than $5 \mathrm{~min}$. Oxidation of quercetin led to form a benzofuranone derivative and several open structures (Sokolová et al., 2016). A dimer of quercetin in acetonitrile was observed only under strict anaerobic conditions (Sokolová et al., 2012). The main peaks of that dimer at $\mathrm{m} / \mathrm{z}$ values are 638, 603, 301, 179, 151, and 109 in negative mode (Sokolová et al., 2012). Furthermore, the dimer is unstable and cannot be detect in the presence of air oxygen. In the current study, the choice of condition in PBS at $4{ }^{\circ} \mathrm{C}$ (less than 5 seconds) and then with $-20{ }^{\circ} \mathrm{C}$ methanol improves the stability of the quercetin dimer and allows its detection. In previous studies, in slightly alkaline conditions at ambient temperature, within 10 min quercetin was oxidized form a benzofuranone derivative and several open structures (Sokolová et al., 2016). However, in current study, during inverting the tube for 5 times, no such quercetin oxidation products could be detected.

\subsection{Myricetin}

When $10 \mu \mathrm{L}$ myricetin was added to $190 \mu \mathrm{L}$ PBS, there was no any obvious change of the color of the solution. However, when $200 \mu \mathrm{L}$ DMSO was added to above solution, the color of solution was turned to blue/black. As shown in Figure 6a, when myricetin was added to PBS, it immediately disappeared and three new compounds were formed which did not happen in 
methanol. UPLC-ESI-MS/MS analysis of these new products reveals main peaks with $\mathrm{m} / \mathrm{z}=469.0385(\mathrm{Rt}=25.01 \mathrm{~min}), 633.0487(\mathrm{Rt}=29.98 \mathrm{~min})$, and $633.0482(\mathrm{Rt}=32.39 \mathrm{~min})(\mathrm{M}+1)$ in positive mode.

The main ion peaks (+ESI) of new product at $\mathrm{Rt}=25.01 \mathrm{~min}(\mathrm{P} 1)$ at $\mathrm{m} / \mathrm{z}$ values are: 451.22055 , 441.24976, 413.19824 (100\%), 395.30405, 315.15240, 287.12106, 277.10419 and 152.89763 (Figure 6b). The main ion peaks $(+\mathrm{ESI})$ of myricetin dimer $1\left(\mathrm{R}_{\mathrm{t}}=29.98 \mathrm{~min}\right)$ at $\mathrm{m} / \mathrm{z}$ values are: 615.30219, 605.27155 (100), 481.33466, 453.13995, 425.16803 and 319.11176 (Figure 6c). The main ion peaks $(+\mathrm{ESI})$ of myricetin dimer $2\left(\mathrm{R}_{\mathrm{t}}=32.39 \mathrm{~min}\right)$ at $\mathrm{m} / \mathrm{z}$ values are: 615.24127 , 605.22491, 481.21344, 453.20233 (100\%), 425.15692, and 319.02109 (Figure 6d). The myricetin dimer 1 and dimer 2 were identified as the dimers of myricetin quinone (Figure 6c an 6d). Myricetin is very unstable and completely disappeared in DMEM within 10 min (Xiao and Högger, 2015b). However, what happened with myricetin is not clear. The dimers of myricetin were hardly ever observed due to their low stability in the presence of air oxygen. We further found that myricetin disappeared in DMEM within less than 5 seconds, and the dimers of myricetin quinone were further oxidized to form new products with $\mathrm{m} / \mathrm{z}=632.06$ (Data not shown here).

\section{Conclusion}

Apigenin is stable when dissolved in with PBS $(\mathrm{pH}=7.4)$ at $4{ }^{\circ} \mathrm{C}$ in air. Kaempferol and baicalein are stable in PBS ( $\mathrm{pH}=7.4)$ at $4{ }^{\circ} \mathrm{C}$ in air with only small amounts of oxidized quinones and hydroxylated products being formed. Flavonols with catechol or pyrogallol groups within 5 seconds converted to their stable dimers and oxidized products in PBS $(\mathrm{pH}=7.4)$ at $4{ }^{\circ} \mathrm{C}$ in the presence of air. PBS ( $\mathrm{pH}=7.4)$ at $4{ }^{\circ} \mathrm{C}$ improves the stability of dimers and allows their detection. The exact mechanism of dimerization, and the bioactivities of the dimers need further investigation. 
The findings reported in this paper may find application in the preservation of foods. Flavonoids are the main protective components against UV damage in apples (Felicetti \& Schrader, 2008; Racsko and Schrader, 2012). Photooxidative sunburn would cause accumulation of oxidized polymeric polyphenols, resulting from scavenging ROS (Rustioni, 2017). And ambient air temperature was identified as the primary causative factor of sunburn damage on apples. PBS $(\mathrm{pH}=7.4)$ under $4{ }^{\circ} \mathrm{C}$ may be applied to cold storage apples to stabilize flavonoids and avoid apple damage. Similarly, the findings may be utilized to control phenolic ripening and tannin oxidation of grapes and strawberries in future (Rustioni et al., 2014; Rustioni et al., 2018; Holzwarth et al., 2012).

\section{References}

Agati G., Azzarello E., Pollastri S., Tattini M. (2012). Flavonoids as antioxidants in plants: Location and functional significance. Plant Sci., 196, 67-76.

Brunetti C., Di Ferdinando M., Fini A., Pollastri S., Tattini M. (2013). Flavonoids as Antioxidants and Developmental Regulators: Relative Significance in Plants and Humans. Int. J. Mol. Sci., $14,3540-3555$.

D'Amelia V., Aversano R., Chiaiese P., Carputo D. (2018). The antioxidant properties of plant flavonoids: their exploitation by molecular plant breeding. Phytochemistry Rev., 17, 611-625.

Cao H., Ou J.Y., Chen L., Zhang Y.B., Szkudelski T., Delmas D., Daglia M., Xiao J.B. (2018). Dietary polyphenols for managing type 2 diabetes: Human studies and clinical trials. Critical Reviews in Food Science and Nutrition, Doi:10.1080/10408398.2018.1492900 
Chen L., Teng H., Jia Z., Battino M., Miron A., Yu Z.L., Cao H., Xiao J.B. (2018a). Intracellular signaling pathways of inflammation modulated by dietary flavonoids: The most recent evidence. Critical Reviews in Food Science and Nutrition, 58, 2908-2924.

Chen L., Teng H., Xie Z.L., Cao H., Cheang W.S., Skalicka-Woniak K., Georgiev M.I., Xiao J.B. (2018b). Modifications of dietary flavonoids towards improved bioactivity: An update on structure-activity relationship. Critical Reviews in Food Science and Nutrition, 58(4), 513-527.

Dangles O., Fargeix G., Dufour C. (1999). One-electron oxidation of quercetin and quercetin derivatives in protic and non protic media. Journal of the Chemical Society, Perkin Transactions 2, 1387-1396.

Felicetti, D. A.; Schrader, L. E. (2008). Photooxidative sunburn of apples: Characterization of a third type of apple sunburn. Int. J. Fruit Sci. 2008, 8(3), 160-172.

Holzwarth, M.; Korhummel, S.; Carle, R.; Kammerer, D. R. (2012). Evaluation of the effects of different freezing and thawing methods on color, polyphenol and ascorbic acid retention in strawberries (Fragaria $\times$ ananassa Duch). Food Res. Int., 48, 241-248.

Li, H., Guo, A., \& Wang, H. (2008). Mechanisms of oxidative browning of wine. Food Chemistry, $108,1-13$.

Molyneux R.J., Waiss Jr. A.C., Haddon W.F. (1970). Oxidative coupling of apigenin. Tetrahedron, 26, $1409-1416$.

Nematollahi D., Malakzadeh M. (2003). Electrochemical oxidation of quercetin in the presence of benzenesulfinic acids. J. Electroanal. Chem., 547, 191-195.

Pham A., Bortolazzo A., White J.B. (2012). Rapid dimerization of quercetin through an oxidative mechanism in the presence of serum albumin decreases its ability to induce cytotoxicity in MDA-MB-231 cells. Biochem. Biophy. Res. Com., 427, 415-420. 
Ramešová S, Sokolová R, Degano I, Bulíčková J, Zabka J, Gál M. (2012). On the stability of the bioactive flavonoids quercetin and luteolin under oxygen-free conditions. Anal. Bioanal. Chem., 402, 975-982.

Ramešová Š., Sokolová R., Tarábek J., Degano I. (2013). The oxidation of luteolin, the natural flavonoid dye. Electrochimica Acta, 110, 646-654.

Ramešová Š., Degano I., Sokolová R. 2017. The oxidative decomposition of natural bioactive compound rhamnetin. J. Electroanal. Chem., 788, 125-130.

Racsko, J.; Schrader, L. E. (2012). Sunburn of apple fruit: Historical background, recent advances and future perspectives. Crit. Rev. Plant Sci., 31, 455-504.

Rustioni, L.; Rocchi, L.; Guffanti, E.; Cola, G.; Failla, O. (2014). Characterization of grape (Vitis vinifera L.) berry sunburn symptoms by reflectance. J. Agric. Food Chem., 62, 3043-3046.

Rustioni, L. (2017). Oxidized polymeric phenolics: Could they be considered photoprotectors? J. Agric. Food Chem., 65, 7843-7846.

Rustioni, L.; Cola, G.; VanderWeide, J.; Murad, P.; Failla, O.; Sabbatini, P. (2018). Utilization of a freeze-thaw treatment to enhance phenolic ripening and tannin oxidation of grape seeds in red (Vitis vinifera L.) cultivars. Food Chem., 259, 139-146.

Sharma H., Kanwal R., Bhaskaran N., Gupta S. (2014). Plant Flavone Apigenin Binds to Nucleic Acid Bases and Reduces Oxidative DNA Damage in Prostate Epithelial Cells. PLoS ONE, 9(3), e91588.

Sokolová R., Degano I., Ramesová S., Bulícková J., Hromadová M., Gál M., Fiedler J., Valásek M. 2011. The oxidation mechanism of the antioxidant quercetin in nonaqueous media. Electrochim. Acta, 56, 7421-7427. 
Sokolová R., Ramešová Š., Degano I., Hromadová M., Gál M., Žabka J. (2012). The oxidation of natural flavonoid quercetin. Chem. Commun., 48(28), 3433-3435.

Sokolová R., Ramešová Š, Kocabova J., Kolivoska V., Degano I., Pitzalis E. (2016). On the difference in decomposition of taxifolin and luteolin vs. fisetin and quercetin in aqueous media; Mon. Chem., 147, 1375-1383.

Takahama U. (1987). Oxidation products of kaempferol by superoxide anion radical. Plant and Cell Physiology, 28, 953-957.

Waterhouse A.L., \& Laurie V.F. 2006. Oxidation of wine phenolics: A critical evaluation and hypotheses. Am J. Enol. Viticul., 57(3), 306-313.

Xiao J.B. 2018. Stability of dietary polyphenols: It's never too late to mend? Food and Chemical Toxicology, 119, 3-5.

Xiao J.B., Högger P. 2015a. Dietary polyphenols and type 2 diabetes: current insights and future perspectives. Current Medicinal Chemistry, 22(1), 23-38.

Xiao J.B., Högger P. 2015b. Stability of dietary polyphenols under the cell culture condition: Avoiding erroneous conclusions. Journal of Agricultural and Food Chemistry, 63(5), 15471557.

Zhao C., Yang C.F., Wai S.T.C., Zhang Y.B., Portillo M.P., Paoli P., Wu Y.J., Cheang W.S., Liu B., Carpéné C., Xiao J.B., Cao H. 2019. Regulation of glucose metabolism by bioactive phytochemicals for the management of type 2 diabetes mellitus. Critical Reviews in Food Science and Nutrition, 59(6), 830-847.

Zhu M., Rajamani S., Kaylor J., Han S., Zhou F.M., and Fink A.L. 2004. The flavonoid baicalein inhibits fibrillation of $\alpha$-synuclein and disaggregates existing fibrils. J. Biol. Chem., 279, 26, 26846-26857. 


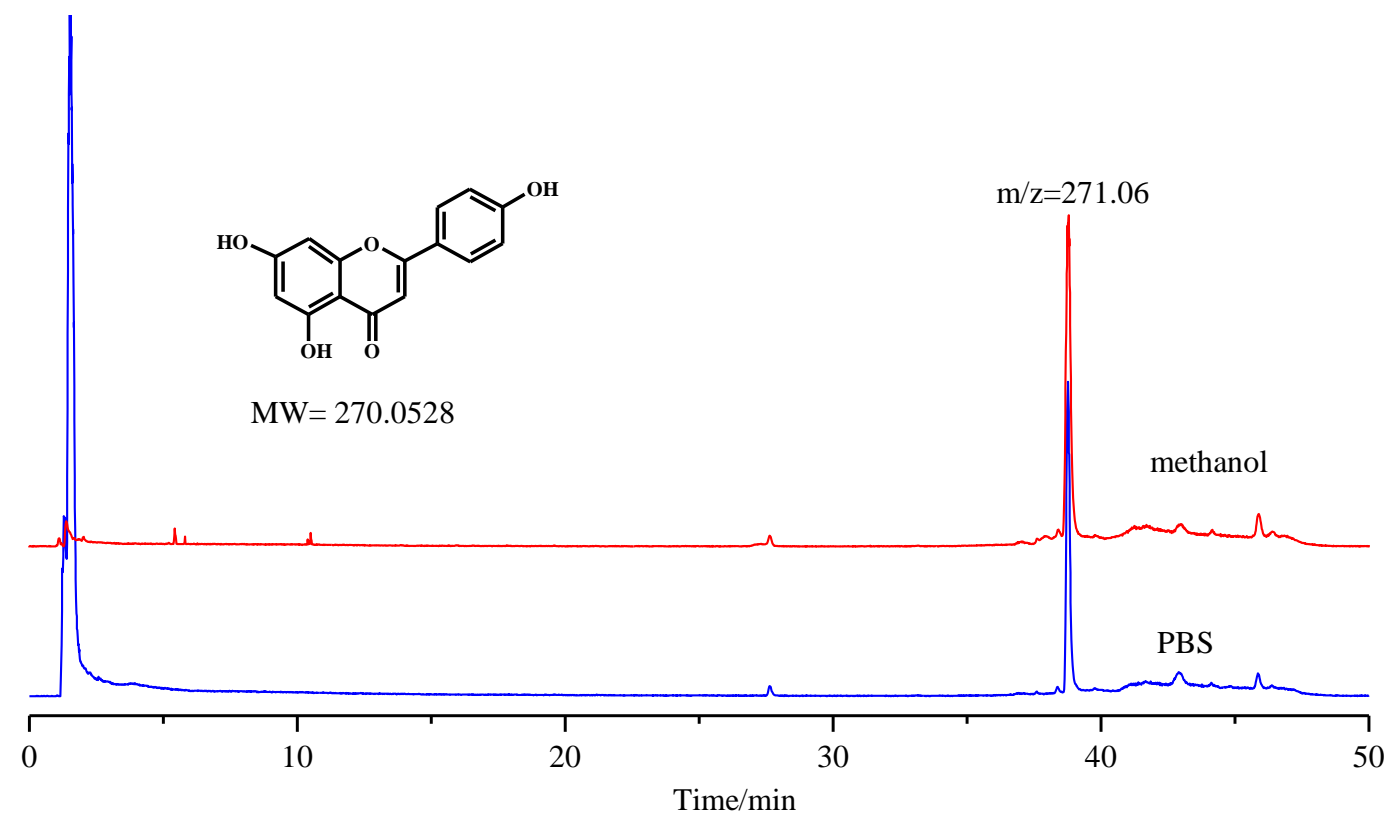

Figure 1 TIC chromatography of apigenin in PBS and methanol (+ESI model). 


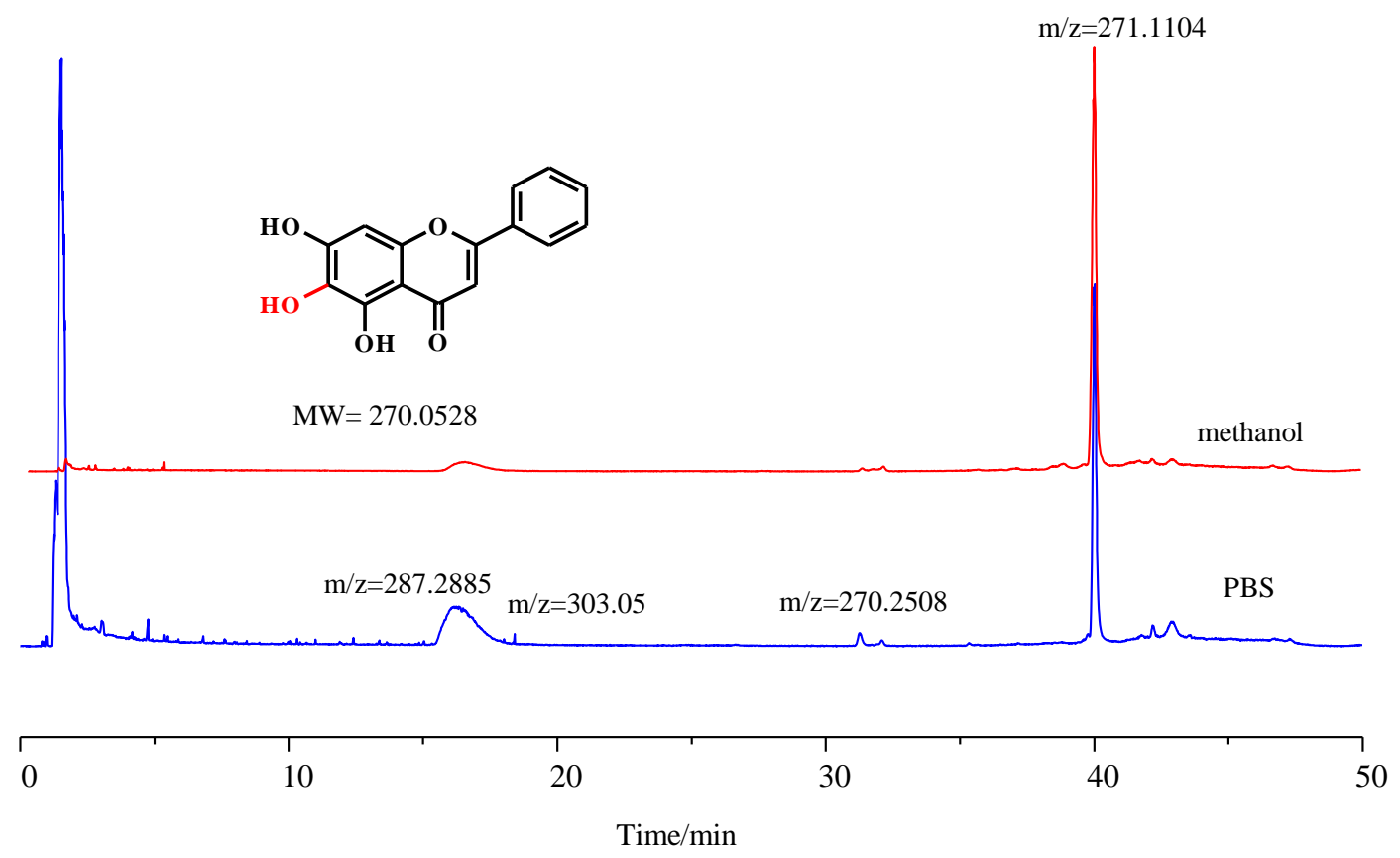

Figure 2. TIC chromatography of baicalein in PBS and methanol (+ESI model). 


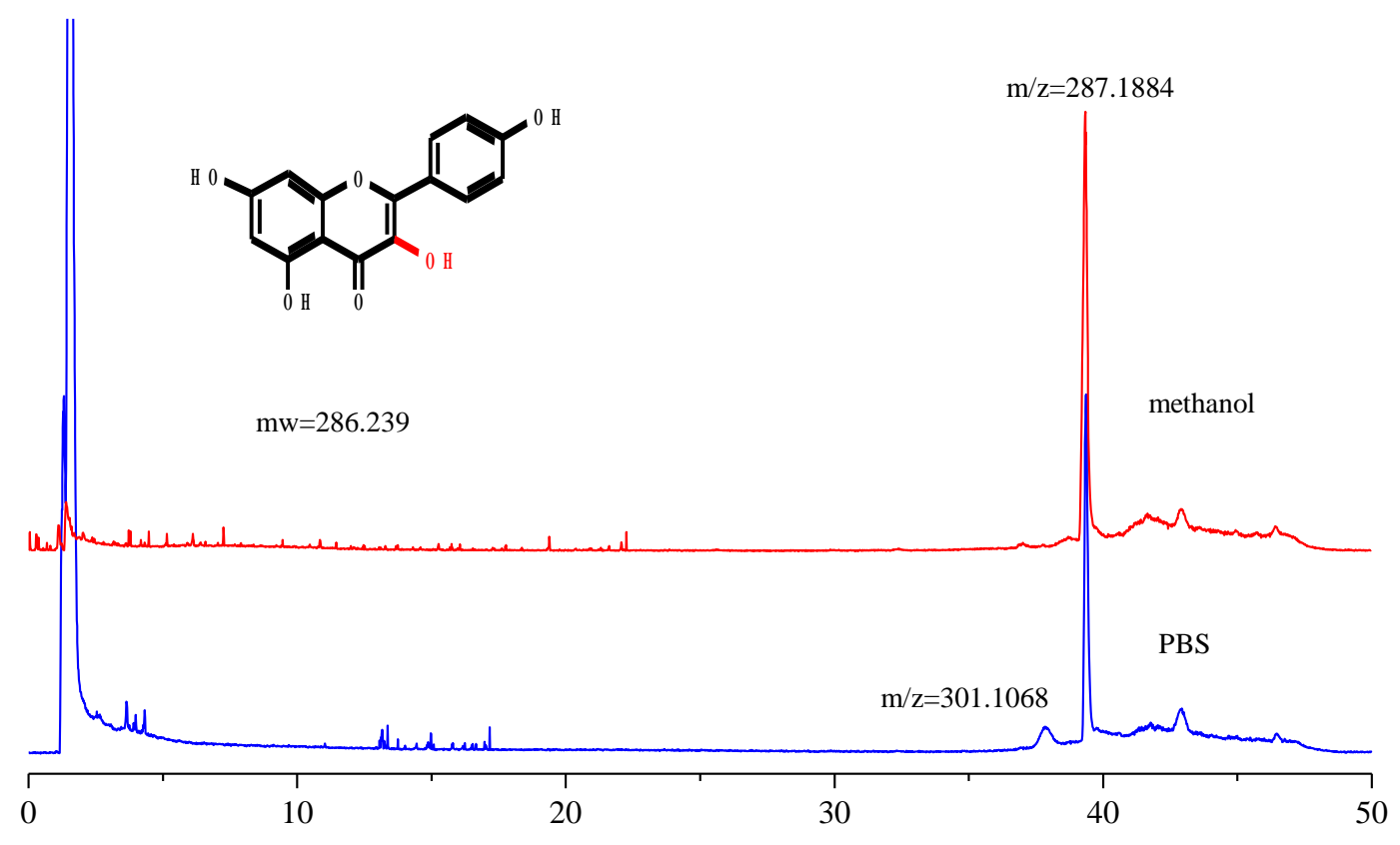

Figure 3. TIC chromatography of kaempferol in PBS and methanol (+ESI model). 


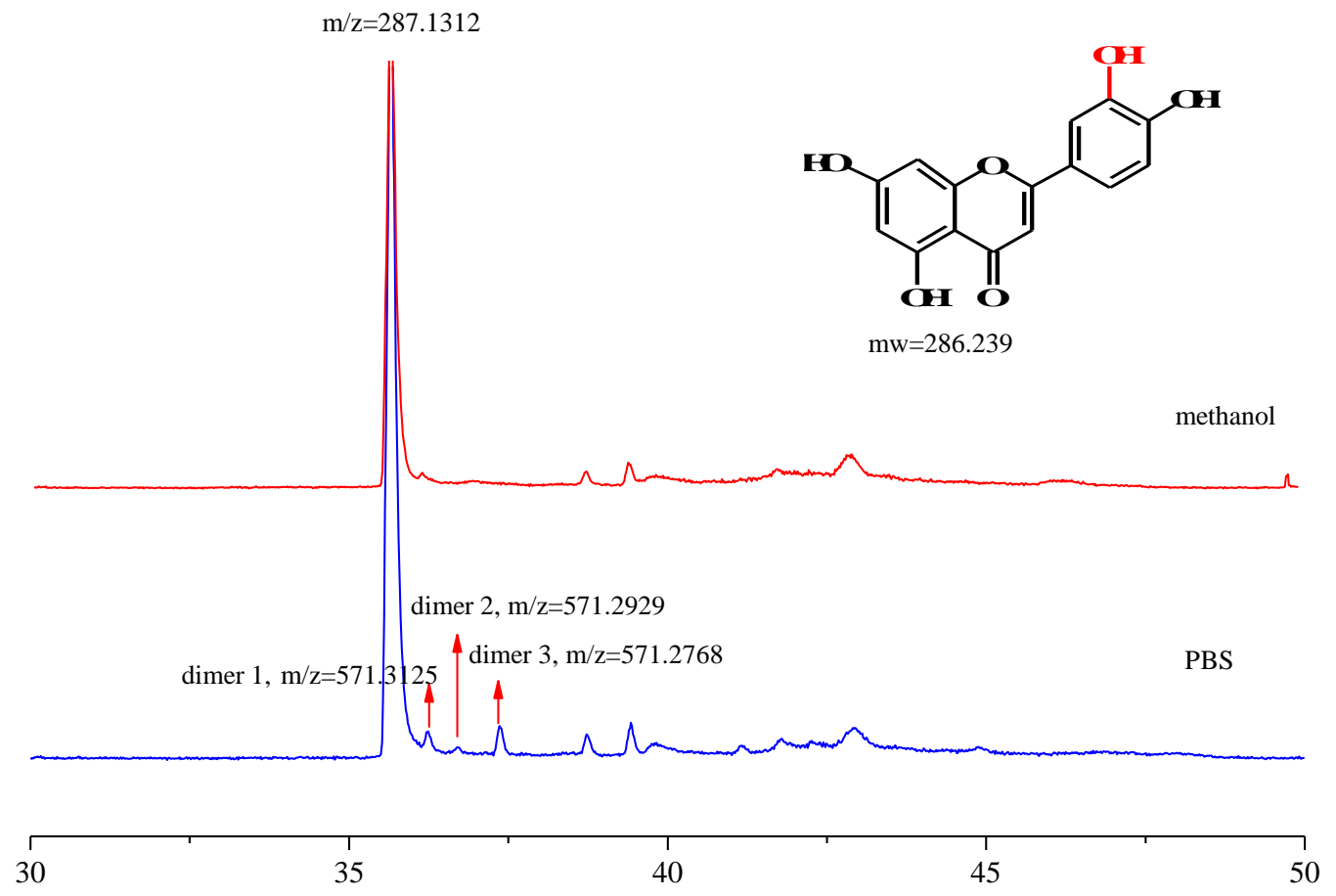

a

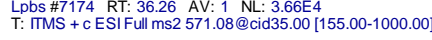

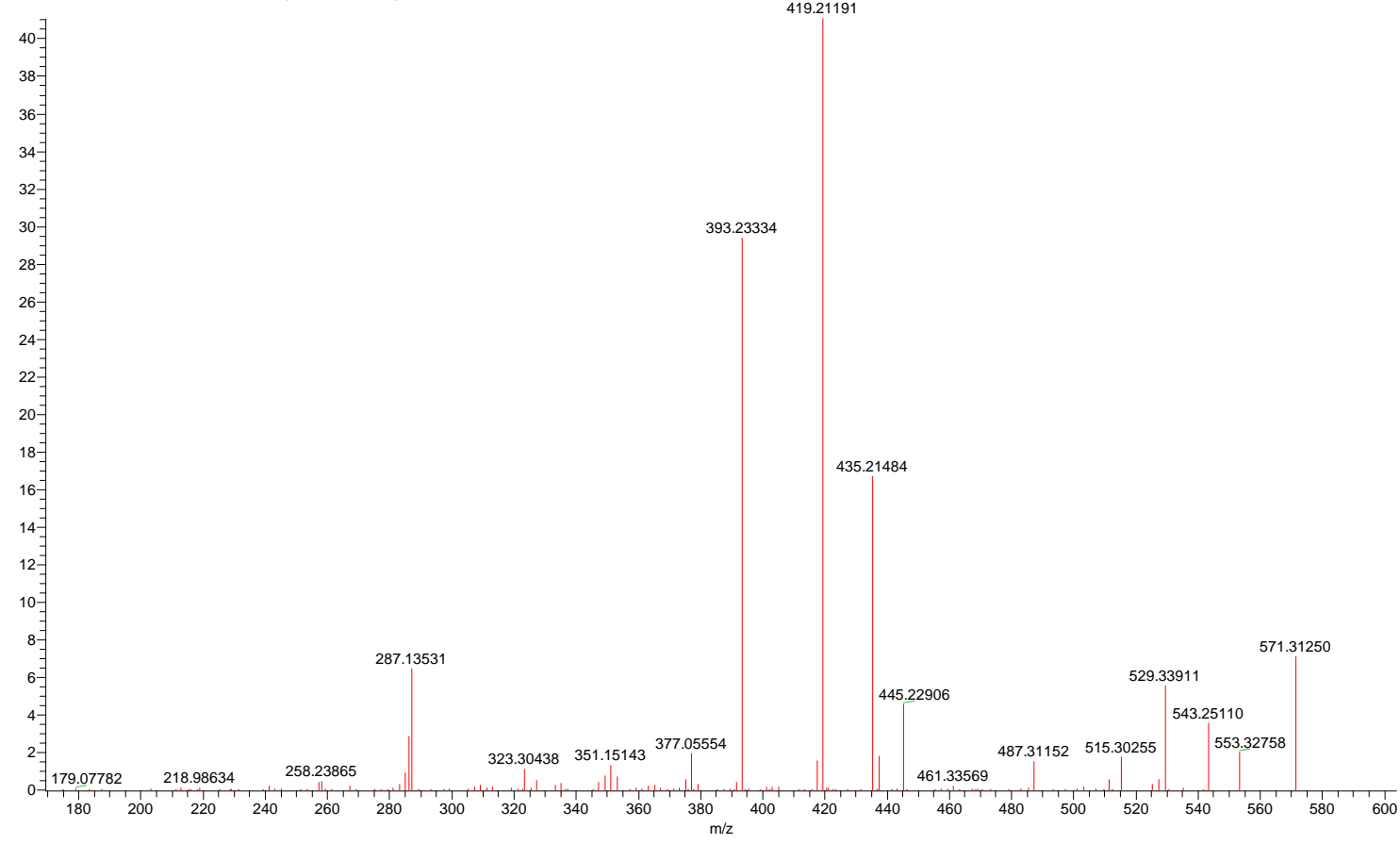



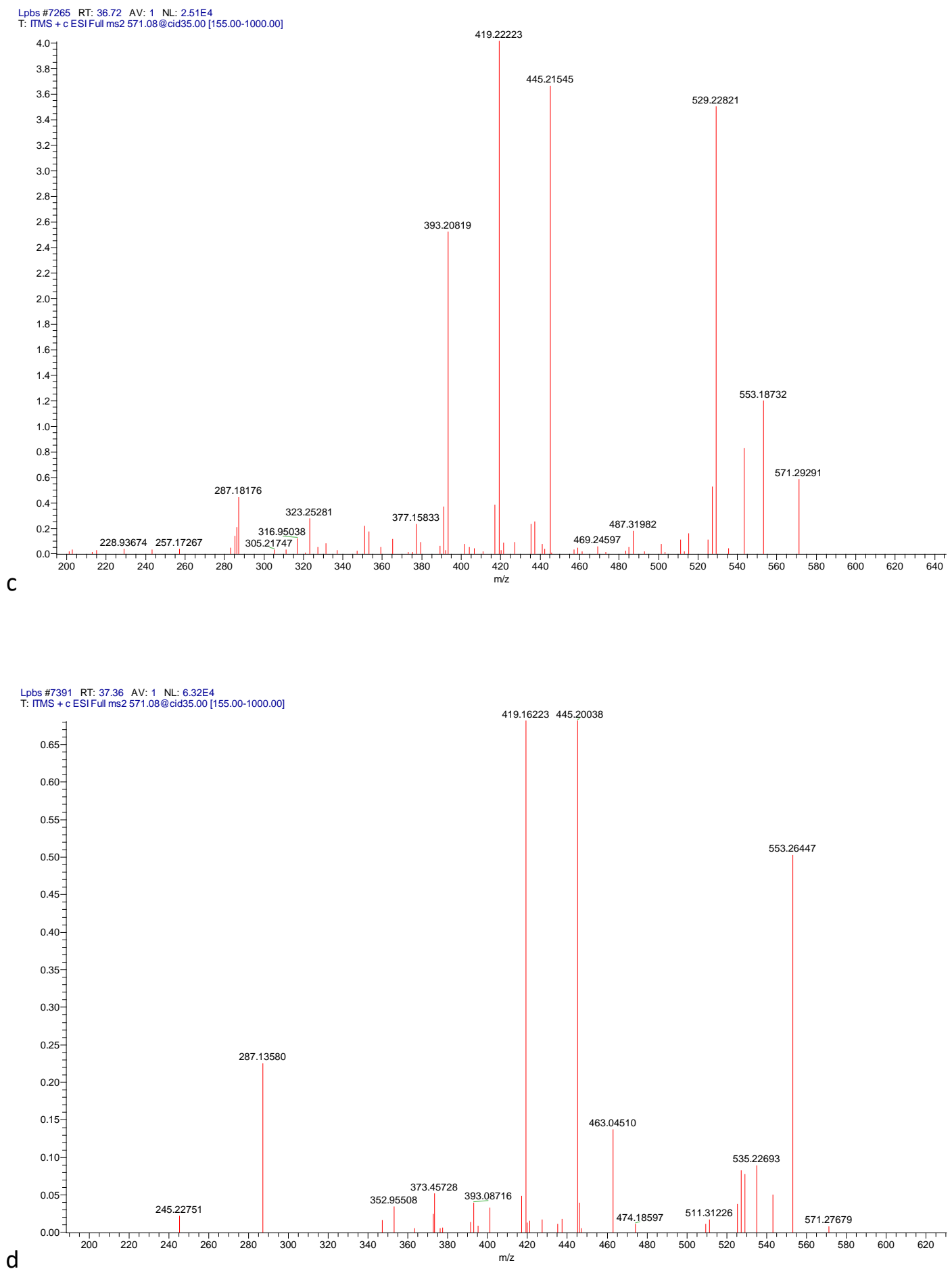

Figure 4. TIC chromatography of luteolin in PBS and methanol (+ESI model) (a); MS/MS of luteolin dimer $1\left(\mathrm{R}_{\mathrm{t}}=36.26\right)(\mathrm{b})$; MS/MS of luteolin dimer $2\left(\mathrm{R}_{\mathrm{t}}=36.72\right)(\mathrm{c})$; MS/MS of luteolin dimer 3 $\left(\mathrm{R}_{\mathrm{t}}=37.36\right)(\mathrm{d})$. 


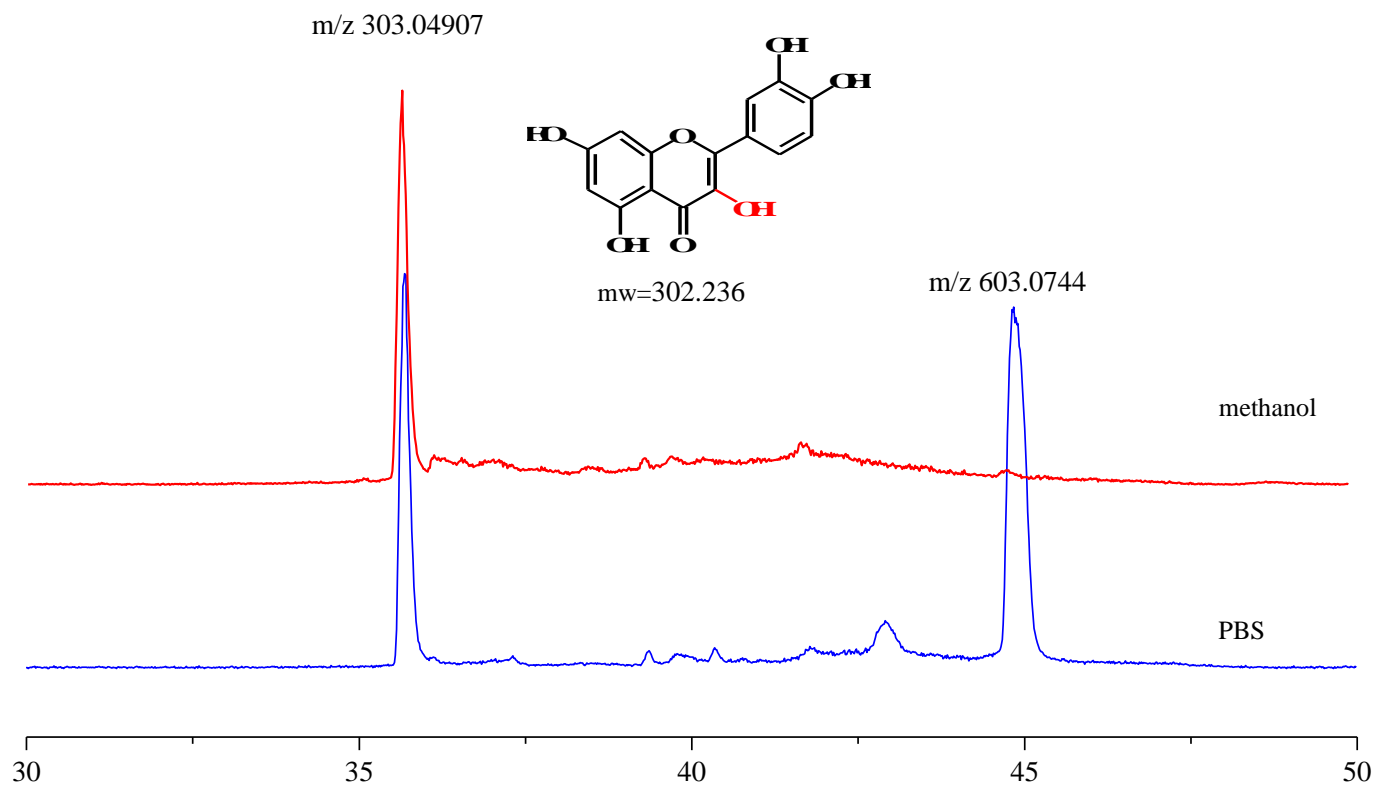

a

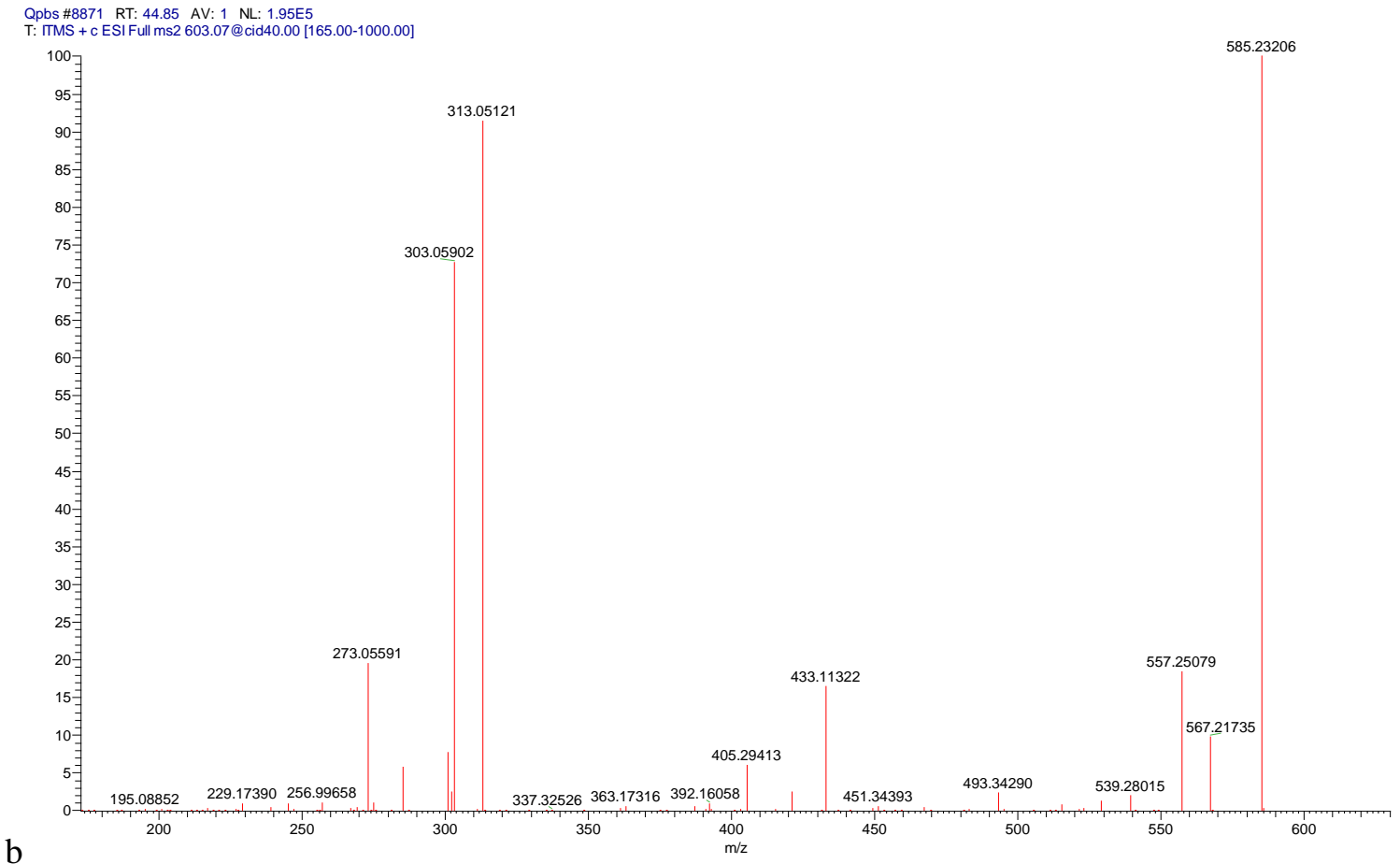

Figure 5. TIC chromatography of quercetin in PBS and methanol (+ESI model) (a); MS/MS of quercetin dimer $1\left(\mathrm{R}_{\mathrm{t}}=44.85\right)(\mathrm{b})$. 


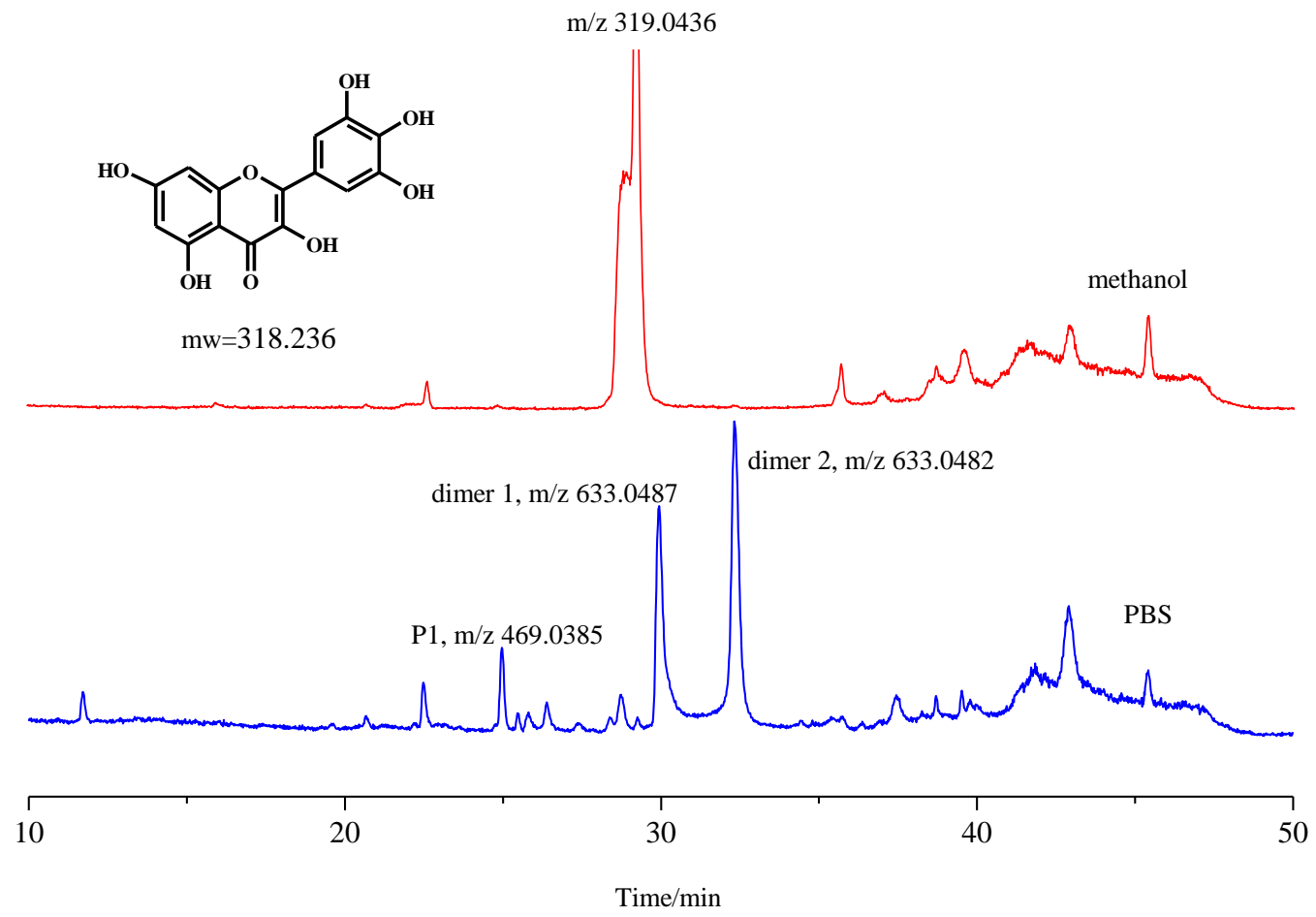

a

Mp \#4829 RT: 25.01 AV: 1 NL: 4.17E3

T: TMS + c ESI Full ms2 469.04@cid35.00 [125.00-1000.00]
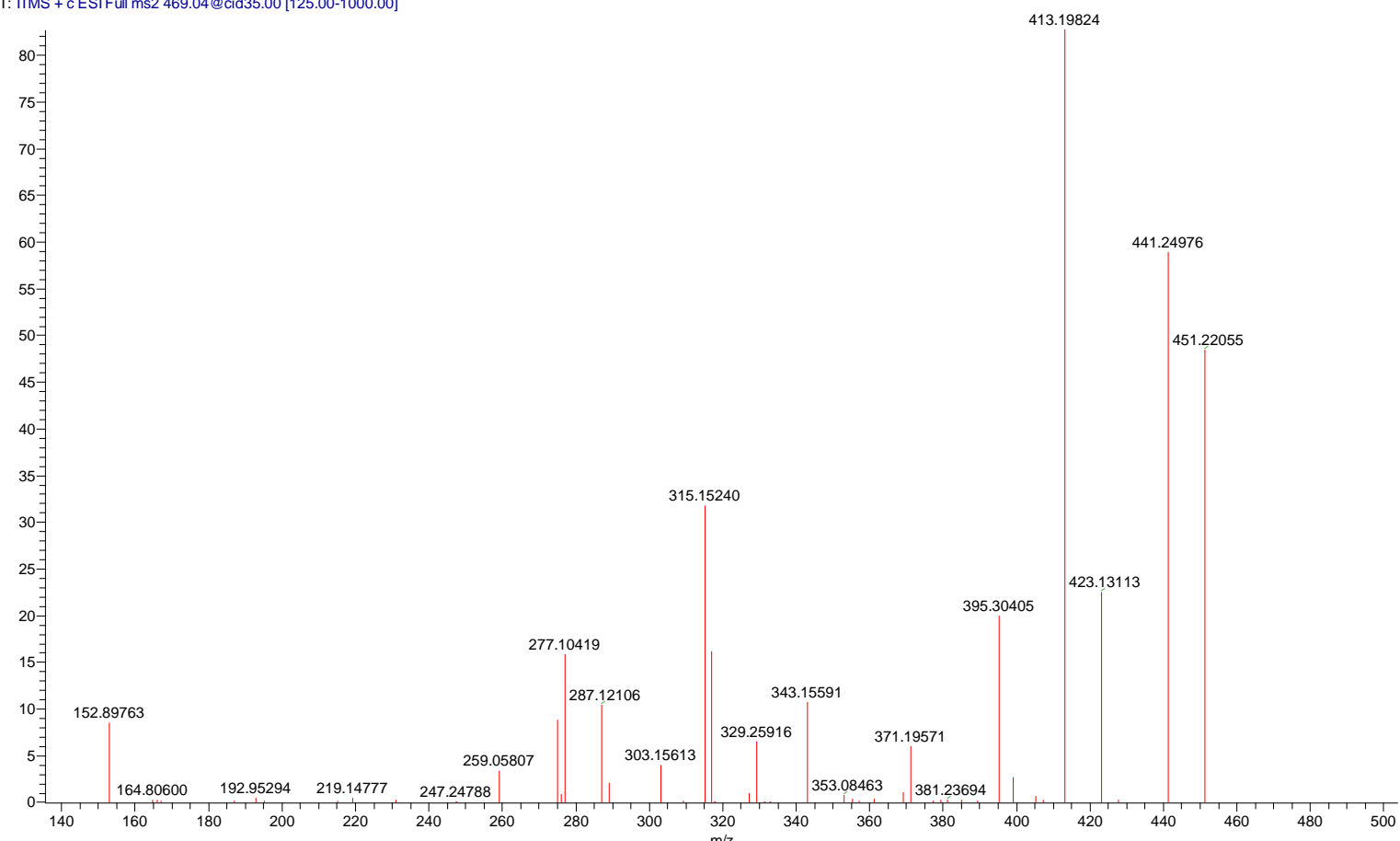

b 


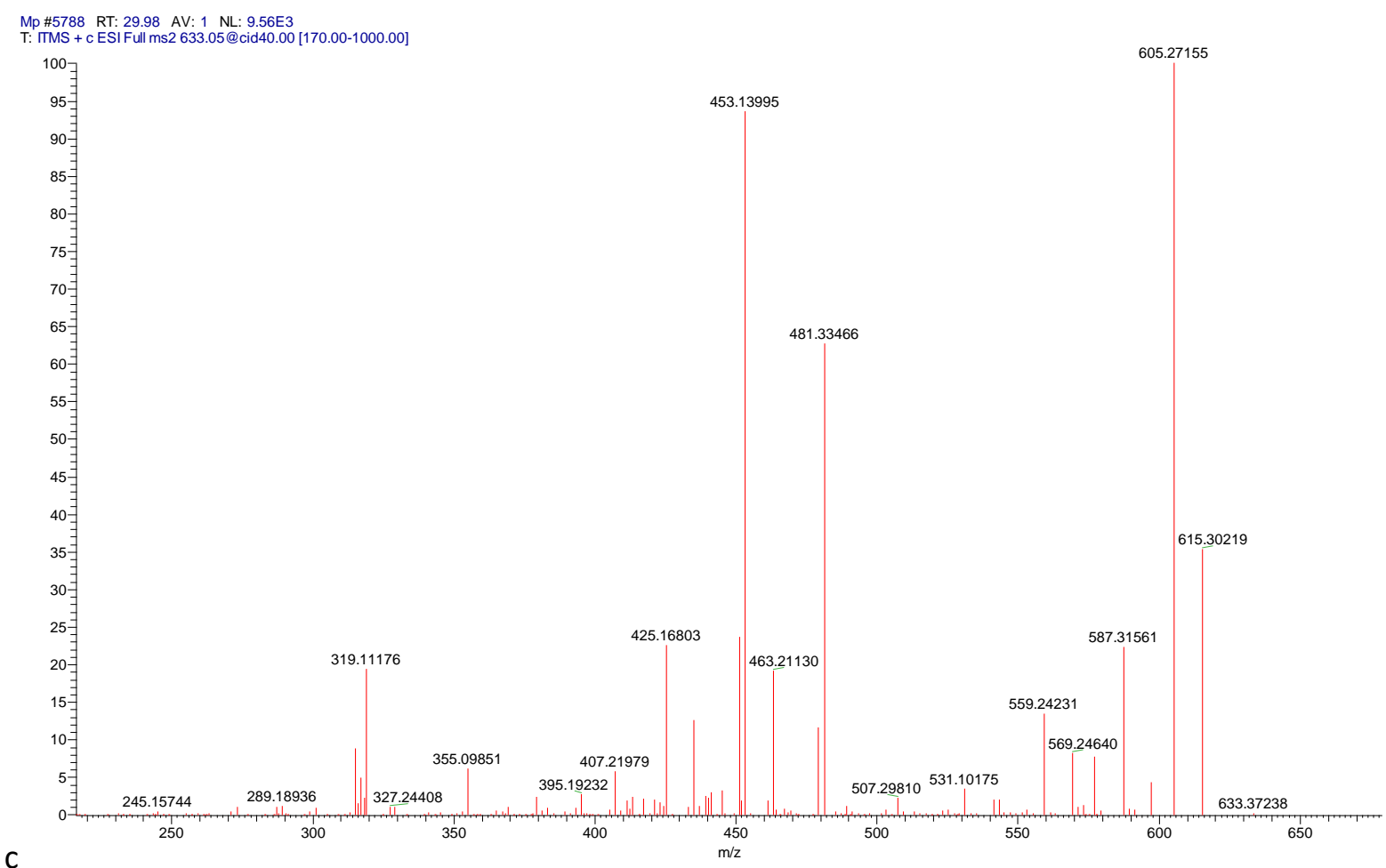

C

Mp \#6253 RT: 32.39 AV: 1 NL: $1.49 E 4$

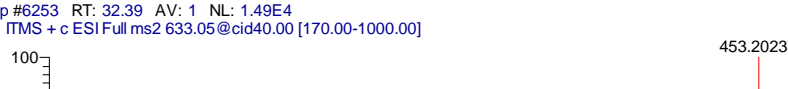

d

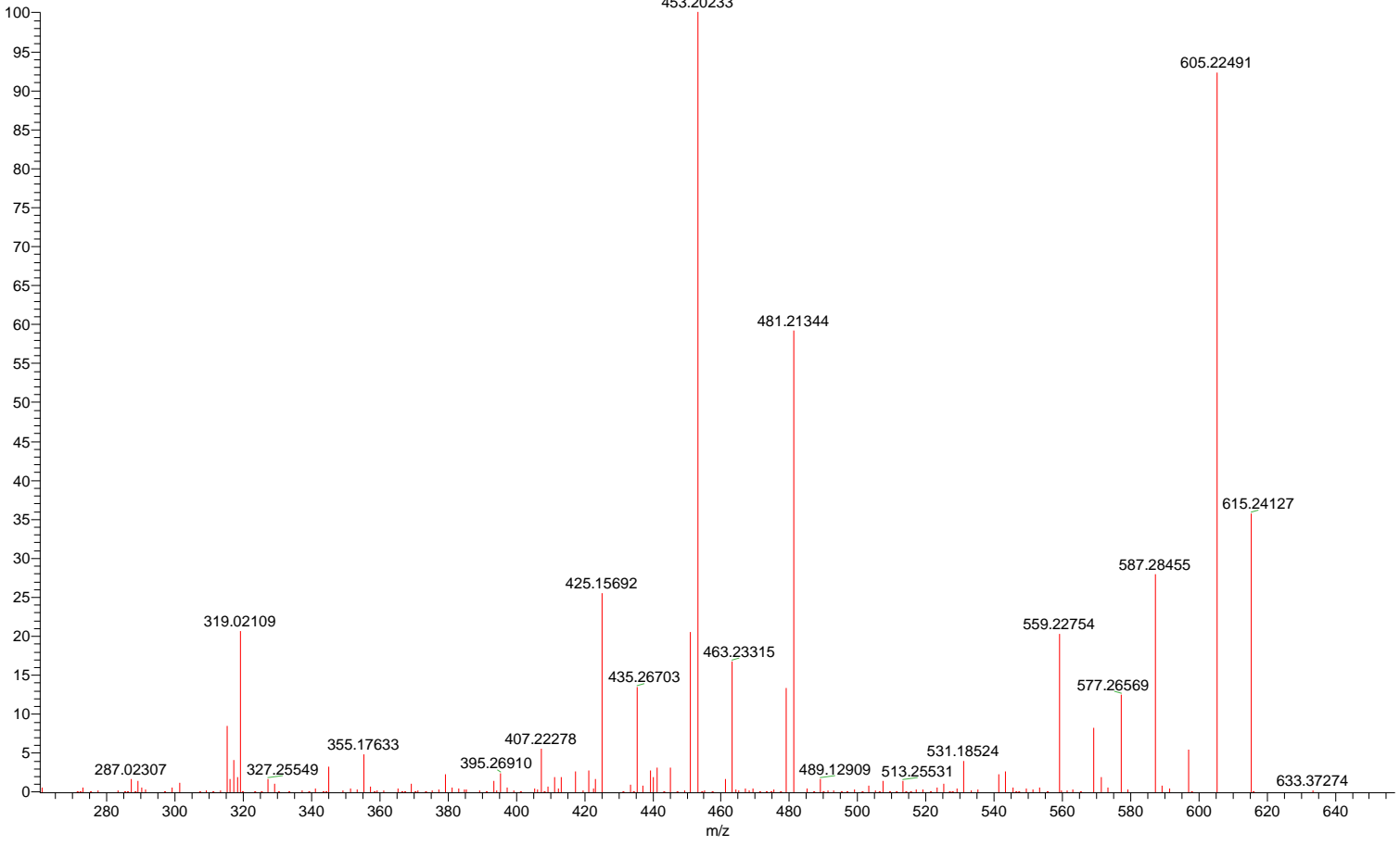

Figure 6. TIC chromatography of myricetin in PBS and methanol (+ESI model) (a); MS/MS of P1 $\left(\mathrm{R}_{\mathrm{t}}=25.01\right)(\mathrm{b})$; MS/MS of myricetin dimer $1\left(\mathrm{R}_{\mathrm{t}}=29.98\right)(\mathrm{c})$; MS/MS of myricetin dimer $2\left(\mathrm{R}_{\mathrm{t}}=32.39\right)(\mathrm{d})$. 